\title{
LE CARCINOME INDIFFÉRENCIÉ DES GLANDES SALIVAIRES
}

\author{
A. CHADLI-DEBBICHE, E. BEN BRAHIM, W. KOUBAÂ, N. LABBÈNE, I. HARIGA*, \\ O. BEN GAMRA *, CH. M'BAREK ${ }^{*}, A$. EL KHEDIM*, M. BEN AYED \\ SERVICE D'ANATOMIE ET DE CYTOLOGIE PATHOLOGIQUES \\ *SERVICE D'OTO-RHINO-LARYNGOLOGIE. \\ HÔPITAL HABIB THAMEUR. TUNIS. TUNISIE
}

\begin{abstract}
Le carcinome indifférencié primitif des glandes salivaires est rare. Son association avec le virus Epstein Barr, initialement décrite chez les esquimaux, est retrouvée dans la majorité des cas publiés. Nous rapportons un nouveau cas tunisien survenu chez un homme âgé de 64 ans, révélé par une tuméfaction de la glande parotide gauche. Microscopiquement se discutait le caractère primitif ou secondaire de la tumeur, étayé par les examens complémentaires. Le patient était traité par une parotidectomie suivie d'un curage ganglionnaire et d'une radiothérapie. L'évolution était favorable après un an de recul.
\end{abstract}

Mots clès : Glande salivaire- Carcinome indifférencié- Virus Epstein Barr

Primary undifferented carcinoma arising in salivary glands is rare. Originally described in Eskimos, the association between Epstein Barr and this tumor is shown by numerous studies.

We report a new Tunisian case occurring in 64-year-old man, revealed by a tumefaction of the left parotid gland. The possibility of metastatic carcinoma was discussed. However, rhinopharyngeal examination and thoracic computerized tomography, failed to show malignancy. Parotidectomy followed by neck dissection and radiotherapy was performed. The patient was alive without recurrence during one year follow-up.

Key words : Salivary gland- Undifferentiated carcinoma- Epstein Barr virus

\section{INTRODUCTION}

Les tumeurs malignes des glandes salivaires sont rares. Elles représentent moins de $1 \%$ de tous les cancers chez l'homme dans le monde, sauf chez les Esquimaux de Groenland (1), du nord du Canada et de l'Alaska ou l'incidence est plus élevée. Dans cette population, plus de $90 \%$ de ces tumeurs répondent à des carcinomes indifférenciés lympho-épithélioma-like (2), évoquant le rôle de facteurs ethniques, géographiques ou comportementaux (3). En Tunisie, le carcinome indifférencié primitif des glandes salivaires est rare: un cas a été rapporté au niveau d'une glande submandibulaire (4). Nous rapportons le deuxième cas et discutons les principales caractéristiques épidémiologiques, l'étiopathogénie et la conduite à adopter devant ce type de tumeur.

\section{OBSERVATION}

II s'agit d'un homme de 70 ans qui présentait une tuméfaction du lobe inférieur de la parotide gauche, de $3 \mathrm{~cm}$ de grand axe, de consistance ferme, à contours polylobés, sans signes inflammatoires en regard. Cette tumeur évoluait depuis 2 ans sans asymétrie faciale.

L'échographie a objectivé une masse parotidienne à double composante tissulaire et kystique de $28 \mathrm{~mm}$ de diamètre. Une parotidectomie exo-faciale était réalisée.
L'examen extemporané était en faveur d'un adénome pleiomorphe. A l'examen anatomopathologique définitif, macroscopiquement la pièce était centrée par un foyer blanc-grisâtre, mal limité et de consistance ferme à la coupe. Microscopiquement, les prélèvements réalisés à ce niveau étaient occupés par une prolifération épithéliale manifestement maligne, agencée en travées ou en plages tumorales (figure 1).

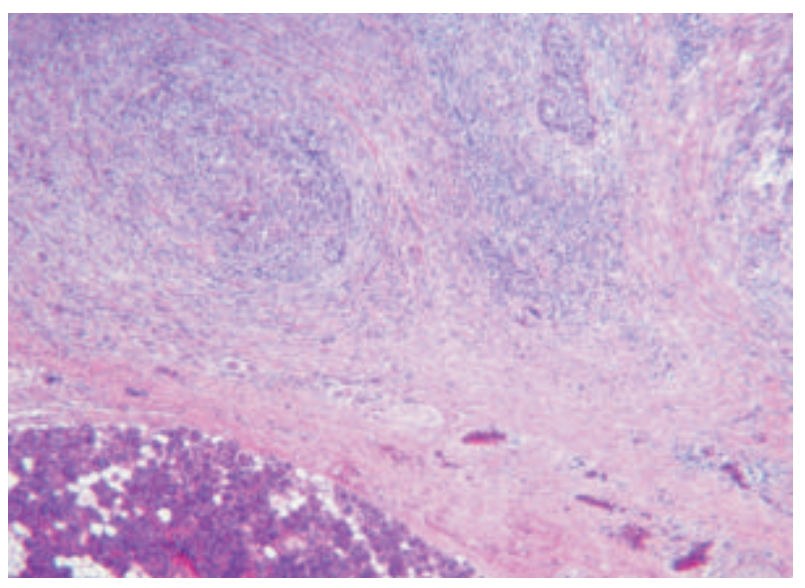

Fig. 1 : Cordons tumoraux dans un stroma fibreux, riche en lymphocytes. En haut, le parenchyme parotidien est d'aspect peu modifié (HEx25). 
Elle était faite de grandes cellules ovoïdes à polygonales aux noyaux volumineux, arrondis ou ovoïdes avec un haut rapport nucléo cytoplasmique et un à deux gros nucléoles centraux. La chromatine était assez claire donnant aux noyaux un aspect vésiculeux. Le cytoplasme était clair, à limites peu visibles, réalisant le classique aspect pseudo-syncitial (figure 2).

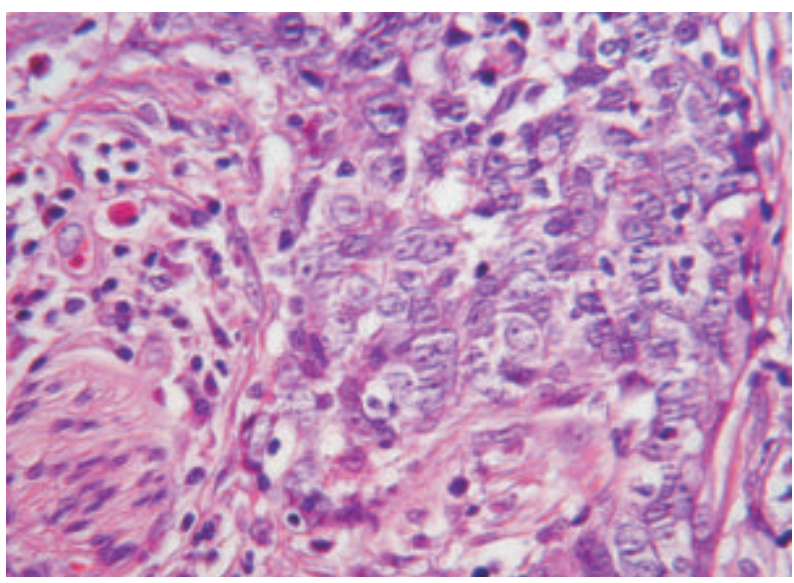

Fig. 2 : Aspect pseudosyncitial des cellules carcinomateuses dont le noyau est volumineux, vésiculeux et pourvu d'un volumineux nucléole (HEx400).

De nombreux lymphocytes étaient présents entre les cellules épithéliales. Le stroma était fibreux et comportait quelques amas de cellules épithélioïdes. Les cellules tumorales exprimaient la cytokératine (figure 3) ainsi que la protéine virale de latence membranaire marquée par l'anticorps monoclonal anti-LMP-1 (figure 4).

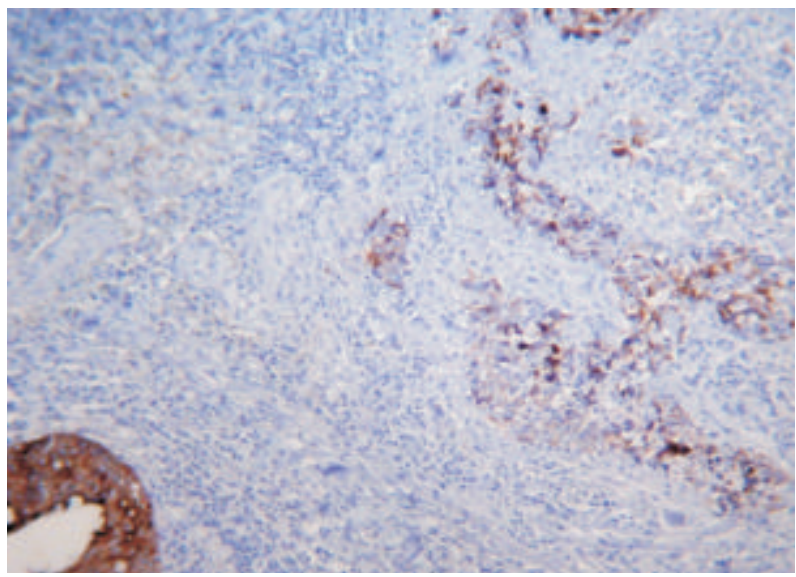

Fig. 3 : Expression de la cytokératine par les cellules tumorales. Un canal excréteur normal est fortement marqué par l'anti-cytokératine (HEx400).

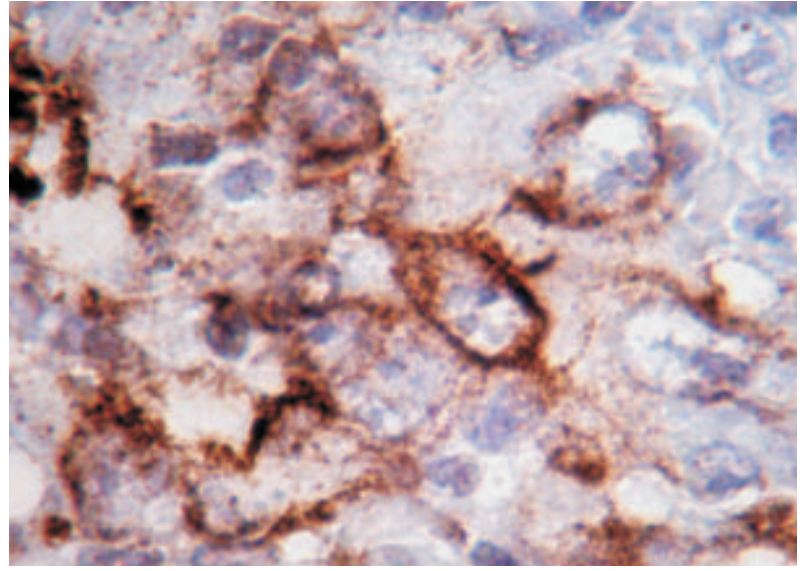

Fig. 4 : Expression des cellules tumorales de la protéine virale de latence membranaire, marquée par l'anticorps monoclonal anti-LMP-1 (HEx400).

Le diagnostic de localisation parotidienne d'un carcinome indifférencié était posé. La tomodensitométrie étant normale et la biopsie du cavum négative, il s'agissait d'un carcinome indifférencié primitif de la parotide. Devant l'apparition d'adénopathies de la chaîne jugulo-carotidienne et rétrospinale gauche, un curage ganglionnaire fonctionnel complet a été réalisé, révélant des métastases à l'histologie. Le malade a bénéficié d'une radiothérapie externe sur la loge parotidienne et les aires ganglionnaires à la dose de 70 Gy. L'évolution était marquée par l'apparition d'une métastase hépatique massive et une altération de l'état général huit mois après la fin de la radiothérapie. Le patient est décédé à 18 mois de l'intervention.

\section{DISCUSSION}

Le carcinome indifférencié primitif des glandes salivaires touche les sujets dont l'âge moyen est de 36 ans pour les femmes et de 44 ans pour les hommes $(2,3,5)$. Une prédominance féminine est rapportée avec un sex-ratio de $1 / 2$ (5). La glande parotide est atteinte dans $90 \%$ des cas chez les esquimaux et les patients d'ethnie blanche. La localisation sous maxillaire est plus fréquente chez les orientaux ou elle est retrouvée dans $1 / 3$ à $1 / 2$ des cas (5). Cliniquement, cette tumeur se manifeste par une masse tumorale indolore, ferme, souvent fixée par rapport au plan superficiel. Macrospiquement, la plupart des tumeurs mesure moins de $5 \mathrm{~cm}$ de grand axe. Ces tumeurs sont partiellement encapsulées, multinodulaires ou infiltrantes. A la tranche de section, les foyers d'hémorragie ou de nécrose sont peu fréquents (5). Le carcinome indifférencié des glandes salivaires est histologiquement et ultrastructuralement semblable au carcinome indifférencié du nasopharynx, également fréquent chez les Esquimaux (1). Une métastase d'un carcinome indifférencié occulte du nasopharynx dans la glande salivaire doit, comme chez notre malade, être éliminée par une endoscopie rhi- 
nopharyngée, une tomodensitométrie et des biopsies étagées du cavum (2).

L'association du virus Epstein-Barr avec ce type de tumeur des glandes salivaires, initialement décrite chez les Esquimaux du Groenland, s'est avérée exister également en Asie, en Europe, en Afrique du nord (4). En effet, à l'immunohistochimie, les cellules tumorales expriment, comme dans notre observation, la LMP-1 et à l'hybridation in situ, il existe des particules virales dans les cellules tumorales et non pas dans les cellules épithéliales de la glande salivaire (5).

Le traitement de choix de cette tumeur est l'association de la chirurgie avec une radiothérapie post-opératoire (3). Les récidives et les métastases ne sont pas rares comme dans notre observation où il existait une atteinte des ganglions de la chaine jugulocarotidienne et rétrospinale gauche. Le taux moyen de survie à 5 ans varie de 70 à $80 \%(3)$.

\section{REFERENCES}

1- Merrick Y., Albeck H., Nielsen NH, Hansen HS. Familial clustering of salivary gland carcinoma in Greenland. Cancer.1986; 57: 2097-102.

2- Sehested M., Hainau B., Albeck H., Nielsen NH, Hansen JPH. Ultrastructural investigation of anaplastic salivary gland carcinomas in Eskimos. Cancer. 1985; 55: $2732-6$.

3- Hanji D., Gohao L. Malignant lymphoepithelial lesions of the salivary glands with anaplastic carcinomatous change: report of nine cases and review of literature. Cancer. 1983; 52: 2245-52.

4- Mrad K., Ben Brahim E., Driss M., Abbes I., Marakchi M., Ben Romdhane K. Lymphoepithelioma-like carcinoma of the submandibular salivary gland associated with Epstein-Barr virus in a North African woman. Virchows Arch. 2004;
445: 419-20.

5- Jang SJ, Paik SS, Lee WM, Park YW, Jang KJ, Tae K et al. Lymphoepithelial carcinoma of the submandibular gland. JKMS. 1997; 12: 252-5.

6- Kuo T, Hsueh C. Lymphoepithelioma-like salivary gland carcinoma in Taiwan: a clinicopathological study of nine cases demonstrating a strong association with Epstein-Barr virus. Histopathology. 1997; 31: 75-82. 